\title{
Mapping the root system of matured trees using ground penetrating radar
}

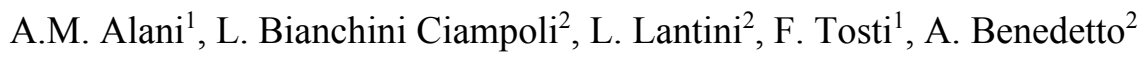 \\ ${ }^{1}$ School of Computing and Engineering, University of West London (UWL) \\ St Mary's Road, Ealing, W5 5RF, London, UK \\ Amir.Alani@uwl.ac.uk; Fabio.Tosti@uwl.ac.uk \\ ${ }^{2}$ Department of Engineering, Roma Tre University \\ Via Vito Volterra 62, 00146 Rome, Italy \\ luca.bianchiniciampoli@uniroma3.it, livia.12910@gmail.com; andrea.benedetto@uniroma3.it
}

\begin{abstract}
This study reports a demonstration of the ground penetrating radar (GPR) potential in health monitoring of tree roots. The main aim of the research was to provide effective and high-resolution mapping of tree root systems. To this purpose, a dedicated data processing methodology, based on two main chronological stages, was developed. First, an algorithm was proposed to filter out the data from noise-related information and to enhance deep reflections from attenuated targets. At a later stage, a multi-step algorithm connecting the identified targets (i.e. the vertices of the reflection hyperbolas) in a three-dimensional environment was created. To demonstrate the viability of the proposed methodology, the soils around two different tree species (i.e. fir and oak trees) were investigated using a ground-coupled multi-frequency GPR system equipped with $600 \mathrm{MHz}$ and 1600 $\mathrm{MHz}$ central frequency antennas. The method has allowed to identify distinctive features in terms of shallow (i.e. within the first $25 \mathrm{~cm}$ from the soil surface) and deep (i.e. lower than $25 \mathrm{~cm}$ from the soil surface) tree root systems for different species of trees.
\end{abstract}

Keywords - tree health monitoring; ground penetrating radar (GPR); tree root system; data processing methodology

\section{INTRODUCTION}

Environmental issues and preservation of natural heritage, especially ancient trees and rare plants, are becoming priority objectives to achieve. Our understanding of how elements of tree health impact each other is limited, often resulting in the quick death of entire forests. Within this framework, the monitoring of tree roots is fundamental to identifying potential damage and early decay. Estimating root depth and spread is a prerequisite for many arboricultural practices, such as tree preservation, and guides a wide range of research decisions [1]. Tree roots develop as a complex system and their classification has historically been based on anatomical or functional features [2]: anatomically, roots can be classified as woody or non-woody [3].

Woody roots are those that have undergone secondary growth, resulting in rigid structure and perennial lifespan. From a functional standpoint, these roots are often referred to as structural roots. Their major role is indeed to anchor the tree and create a framework for the root system. The zone of rapid root taper is often identified as the area within $1-2 \mathrm{~m}$ of the trunk on larger trees. Structural roots found in this area frequently exhibit considerable secondary thickening, which is not present on roots farther from the trunk [4]. Near the trunk juncture, structural roots on large trees may become thickened eccentrically along the vertical plane. Hence these are referred to as buttress roots, reflecting their shape and stabilisation function. Beyond the zone of rapid taper emanates a framework of woody structural roots that provide additional anchorage and serve as conduits for the long-distance transport of water, nutrients, and metabolites. The size of these roots may be influenced by mechanical stresses, with more large roots forming in the windward and leeward directions in trees subjected to winds from one direction [5].

Non-woody roots are so named for not developing secondary growth and for proliferating from the structural root framework. These roots form the extensive network that develops across the root surface area. Their main function is the uptake of water and nutrients; hence they are frequently referred to as fine or absorbing roots. Non-woody roots have generally small diameters $(<2 \mathrm{~mm})$, high metabolic rates and a lifespan ranging from a few days to weeks $[6,7]$. Despite the above diminutive size, fine roots can account for as much as $90 \%$ of the total root system length [8].

Root system health is strictly related to the soil environment. Aside from serving as a substrate for anchorage, the soil provides water, minerals, and oxygen, all of which are essential for root development. To that effect, root function and development can be severely impaired in soils where these resources are limited. Root growth is commonly limited by drought, waterlogging, hypoxia, soil compaction, salinity and high concentrations of heavy metals $[9,10]$.

In this complex subsurface environment, it is necessary to identify technologies and equipment for effective detection and mapping of tree roots. Destructive methods allow a description of the root system at the time point of sampling, therefore they are of limited value for investigating the development processes. Moreover, these techniques are intrusive to the area of investigation and they might also affect the surrounding environment [11]. On the other hand, the use of non-destructive testing (NDT) methods in preserving natural heritage, especially ancient trees, has increased in recent years. Across the available NDTs, the ground penetrating radar (GPR) technology has 
proved to be one of the most effective tools, due to a high versatility, rapidity in data collection and the provision of reliable results at relatively limited costs. There exist extensive applications of GPR in soil sciences [12-14] aimed at assessing main physical properties of the soil (e.g. $[15,16])$. Applications of GPR in forestry science [17] are related - but not limited to effective tree trunk assessments and appraisals, tree root mapping, and soil interaction with trees and plants. In regard to the investigation of tree root systems, research was addressed towards the assessment of root diameter [18], biomass [19], coarseness [20] and root patterns in urban areas [21, 22]. For the assessment of tree trunks, factors such as the inner structure [23], the anisotropy (grain orientation) [24], water content [25] and the frequency of the applied electromagnetic (EM) field [26] were mostly analysed. More recently, numerical simulations of the GPR signal have been used for the interpretation of the geometric and physical properties and decay processes of trunks $[27,28]$.

\section{AIM AND OBJECTIVE}

The main aim of the research was to provide an effective and high-resolution mapping of the tree roots.

To achieve this aim, the main objective was to develop a data-processing methodology for achieving an effective threedimensional (3-D) visualisation of shallow and deep tree root systems.

\section{Methodology}

Two main chronological data-processing stages were developed. A pre-processing algorithm for signal noise filtering and amplification of attenuated targets was first produced to aid operators and decision-makers with the interpretation of better quality GPR images. Hence, the algorithm for the identification of tree roots and sequential tracking in a 3-D domain was developed.

\section{A. Pre-Processing Algorithm}

The motivation for this processing stage stems from the need to reduce clutter in GPR data. Moreover, doable data analysis and interpretation are needed for extracting quantifiable EM wave properties to be translated into application-specific information. With the signal-processing area being so extensive and each processing method being so purpose-specific, it was decided to adopt a range of standard signal-processing filters and functions. To this effect, the pre-processing algorithm was made applicable to different types of data, thereby lowering the risk to achieve distorted information by an over-processing of data [29]. Sequential use of a) zero-offset removal, b) background removal, c) bandpass filtering and d) time-varying gain was carried out.

1) Zero-offset removal: This process operation was applied to ensure that the mean value of the A-scan was near to zero [30]. To solve potential wow effects, a simple averagesubtraction algorithm was used. The result of this algorithm application was an A-scan with a mean equal to zero, that is a symmetric probability distribution of amplitude along the Ascan.
2) Background removal: This filter applied to B-scan data, unlike all the other techniques involved in the proposed preprocessing algorithm. It allowed to enhance the reflection peaks - given by the presence of roots - by eliminating the background noise.

3) Bandpass filtering: the aim of this process was to filter real data by excluding signal components outside the main frequency range. In more detail, the Butterworth filter was applied here among the available set of bandpass filters. To avoid the cutting of frequency regions including relevant information, the frequency range was centred around the nominal frequency of the antenna. In addtion, the width of the pass band was set 1.5 times the central frequency (e.g. [31]).

4) Time-varying gain: A time-varying gain function was applied to compensate for the rapid fall off in radar signal propagation due to increasing depths and/or highly conductive materials. The application of time gain to radar data may vary according to the target of the processing. In this study, a spherical and exponential gain compensation (SEC) has been applied [31]. It is worth mentioning that use of this function severely alters the original shape of the signal; hence artifacts might be generated, along with incorrect data interpretation. Fig. 1 shows an example of time-varying function application from $1 \mathrm{~m}$ below the ground surface. Attenuated hypebolas in Fig. 1(a) turn out to be clearer in Fig. 1(b). However, background noise - unuseful for the purposes of the investigation - is also highlighted. Therefore, in order to minimise data alteration, the time-varying gain function was applied as the last stage of the pre-processing algorithm.

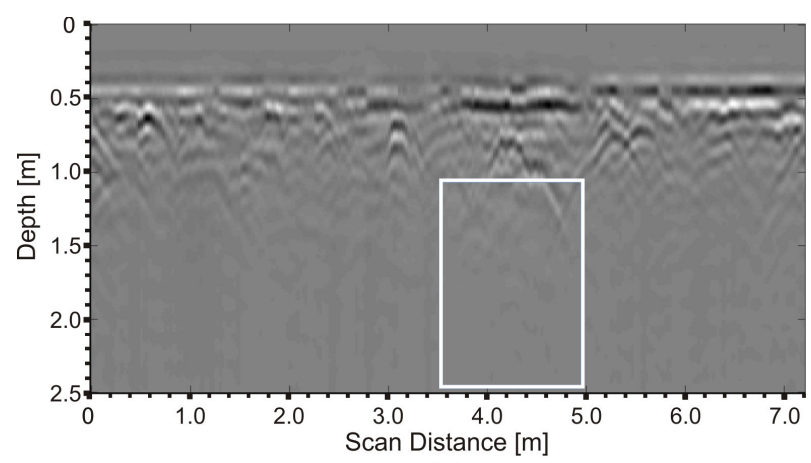

(a)

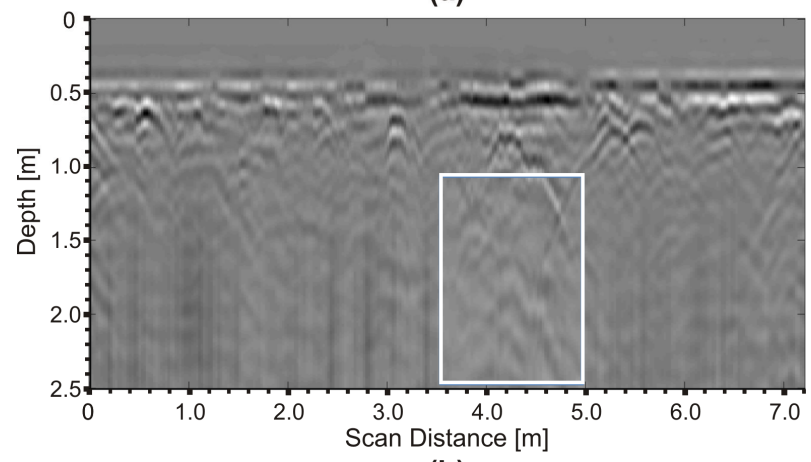

(b)

Fig. 1. B-scan before (a) and after (b) the application of the time-varying SEC gain function. 


\section{B. Tree Root Tracking Algorithm}

A multi-stage methodology was developed for the algorithm. First, the preliminary assumptions (i.e. data acquisition method and the dielectric properties of the medium) and data input (i.e. the outcomes of pre-processing algorithm, matrix dimensions, GPR data acquisition settings) were defined.

Afterwards, an iterative processing method was developed, based on the comparison of the amplitude values in a given position of the 3-D domain with a threshold. Finally, the output rendering and its conditions were produced and discussed.

1) Preliminary assumptions: The proposed model is based on two main assumptions, including:

- The data acquisition method (i.e. longitudinal or circular transects).

- The dielectric properties of the investigated medium.

In regard to the acquisition method, it is worth mentioning that GPR tests carried out with circular transects (i.e. by rotating around the tree with constant radial distance - Fig. 2(a)) should likely ensure a quasi-perpendicular scanning of the root systems. Hence, this turns out to detect tree roots with a clearer hyperbolic pattern. In view of the above, the algorithm has been developed with reference to a three-dimensional system of cylindrical coordinates (Fig. 2(b)), in which the vertical axis coincides with the direction identified by the axis of the tree trunk and the origin is placed at the point where the axis intersects the horizontal plane (i.e. where the trunk ends and the root system begins). The system is identified by the depth $z$, the angular coordinate $\theta$ and the radial coordinate $\rho$, which represents the distance from the centre of the tree. To make the methodology more flexible to the context of application (e.g., a survey where radial scanning is not feasible), implementation of the algorithm was also allowed in case of acquisition of longitudinal transects.

The relative dielectric permittivity of the medium was assumed to be homogeneous throughout the investigation area and was estimated according to the following relationship:

$$
\varepsilon_{r}=\left(\frac{c_{0}}{v}\right)^{2}
$$

where $\varepsilon_{r}$ is the relative permittivity of the medium, $c_{0}$ is the speed of light in free space and $v$ is the wave propagation speed in the medium.

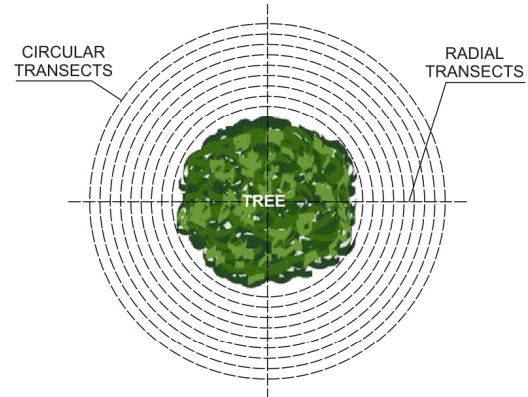

(a)

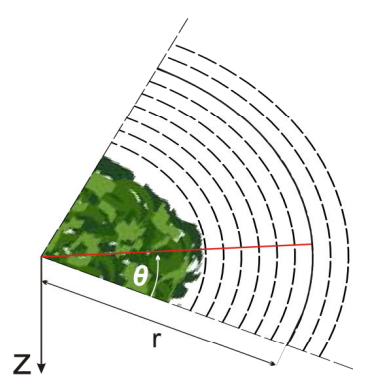

(b)
Fig. 2. Data acquisition framework with cylindrical coordinates. Circular transects' scheme (a) and 3-D system of cylindrical coordinates (b).
The location in depth $s_{i}$ of the $i^{\text {th }}$ hyperbola vertex from the ground surface was obtained as follows:

$$
s_{i}=\frac{\Delta t_{i}}{2} \cdot v
$$

where $\Delta t_{i}$ is the $i^{\text {th }}$ two-way travel time taken by the EM wave to cover the distance from the transmitter to the receiver after being reflected by the target. The value of $v$ was taken as the average value of velocities estimated by hyperbola fitting [32] of several roots' reflection patterns across the whole investigation area.

2) Data input: The algorithm receives GPR data from the pre-processing stage, in the form of a three-dimensional matrix of real numbers $A(I, J, K)$, composed of signal amplitude values in a generic point $i, j, k$. The index $i$ corresponds to the number of GPR scans, limited to $I$, the index $j$ corresponds to the scan direction, limited to $J$, and $k$ is the vertical coordinate going into the ground, limited to $K$. In the case of acquisitions with circular transects, the coordinates of a generic point $(i, j, k)$ will be achieved from the following expressions:

$$
\begin{gathered}
x=\rho(i) \cos \theta(j) \\
y=\rho(i) \sin \theta(j) \\
z=z(k)
\end{gathered}
$$

3) Iterative procedure: The aforementioned assumptions and inputs are the basic information for the development of an iterative procedure for root system tracking. A multi-stage process was developed as follows:

a) Target identification: The root is identified by locating the vertex of a reflection hyperbola. Amplitude values not likely related to the tree roots are filtered out from the 3-D matrix.

b) Correlation analysis (3-D domain): Further hyperbolas' vertices are investigated in the neighbourhood of the one identified in the previous step.

c) Tracking of continuous segments: The algorithm follows the hyperbolas' vertices, creating a vector that corresponds to a single root.

d) Reconstruction of root system geometry: The vectors created in the previous step are positioned into a 3-D rendering of the tree root system.

e) Evaluation of root diameters: Starting from the 3-D rendering, roots are further analysed to investigate the possibility of evaluating their size (e.g. [33]) and to visualise this feature into the rendering.

\section{EQUIPMENT AND FIELD SURVEYS}

A ground-coupled multi-frequency GPR system equipped with $600 \mathrm{MHz}$ and $1600 \mathrm{MHz}$ central frequency antennas was used for testing purposes. Data were acquired using a time window of $40 \mathrm{~ns}$ and 512 samples. The horizontal resolution was set to $2.4 \times 10^{-2} \mathrm{~m}$.

In order to reach the maximum penetration depth of the root system, only the $600 \mathrm{MHz}$ frequency was considered for data processing purposes. 
Two main surveys were carried out to verify the viability of the proposed multi-stage algorithm for different species of trees.

A first survey focused on the investigation of the soil around a 70 -year-old fir tree, with a trunk circumference of $3.40 \mathrm{~m}$ and an average radius of $0.55 \mathrm{~m}$. A number of 9 circular transects, at $0.30 \mathrm{~m}$ intervals, were carried out all around the tree circumference starting at $0.50 \mathrm{~m}$ from the outer surface of the bark (Fig. 3(a)).

A second survey took place on the soil around an isolated oak with a trunk circumference of $2.48 \mathrm{~m}$ and an average radius of $0.39 \mathrm{~m} ; 10$ scans, located $0.30 \mathrm{~m}$ from one another, were carried out. A semi-circular configuration was chosen for this survey. The inner scan started at $0.60 \mathrm{~m}$ from the outer surface of the bark, while the outer one had a radius of $3.69 \mathrm{~m}$ (Fig. 3(b)).

\section{RESULTS AND SHORT DISCUSSION}

\section{A. Fir Tree Investigation}

The application of the pre-processing algorithm to the raw data has allowed to enhance the visualisation of the reflection hyperbolas. Fig. 4 shows the application of the time-varying gain function to the innermost circular scan $(r=1.05)$. As can be seen from the common rectangular windows, the gain function improved the display of the deeper targets (Fig. 4(b)), which were hidden by signal attenuation (Fig. 4(a)). To avoid the formation of artifacts, it was decided to apply gain up to $k=250$ (i.e. depth of $195 \mathrm{~cm}$ ).

The tree root tracking algorithm was then executed on the pre-processed data. In order to aid in the interpretation of the results, it was decided to identify shallow (within the first $25 \mathrm{~cm}$ from the soil surface) and deep (i.e. below $25 \mathrm{~cm}$ from the soil surface) roots with different colours in the graphs. Fig. 5 shows a two-dimensional (2-D) plan view (Fig. 5(a)) and a 3-D rendering (Fig. 5(b)) of the tree root system of the investigated fir tree.

It can be observed that the algorithm was capable of finding paths compatible with the trend of the roots', identifying relevant elements connected to one another according to the logic-based and signal-noise-based instructions. For sake of consistency, the coordinates of the points were double-checked with the positions of the hyperbola apices detected in the collected B-scans.

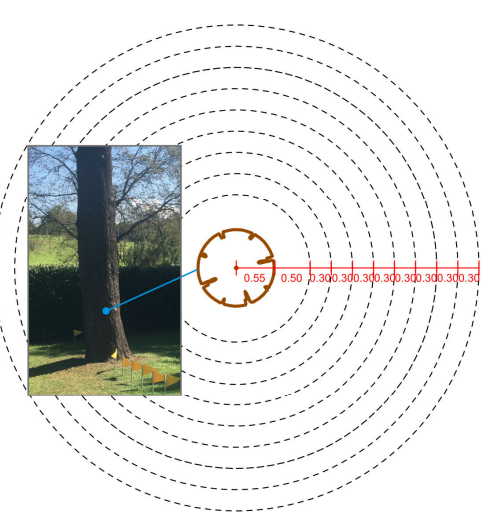

(a)

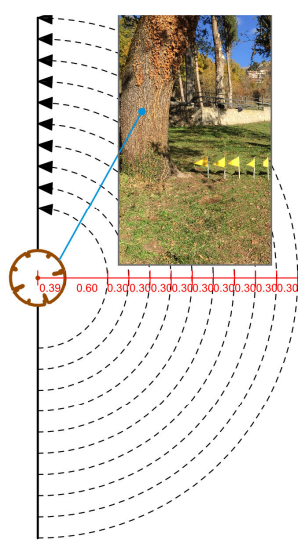

(b)
Fig. 3. Survey schemes for the GPR data acquisitions: circular configuration (fir tree) (a) and semi-circular configuration (oak tree) (b).

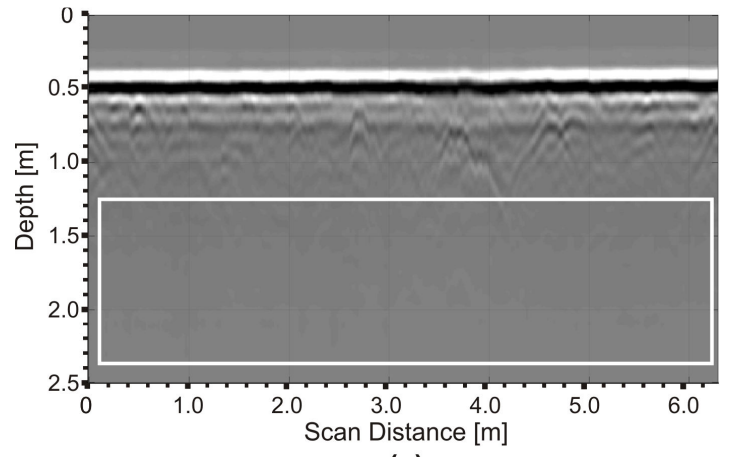

(a)

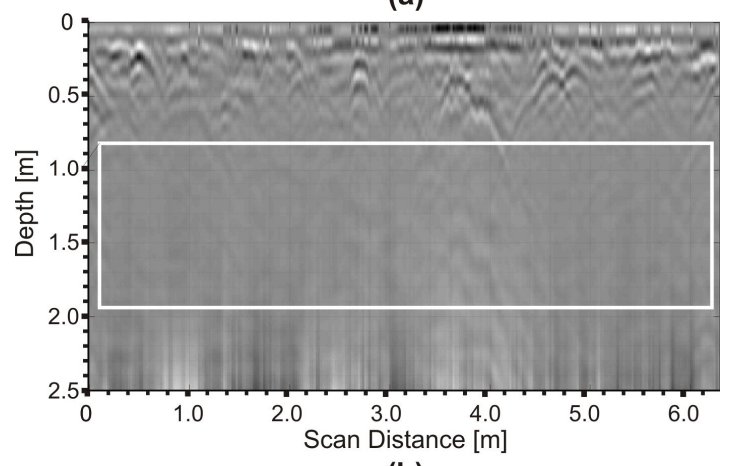

(b)

Fig. 4. B-scan collected in the fir tree survey before (a) and after (b) the application of the pre-processing stage with time-varying SEC gain function.

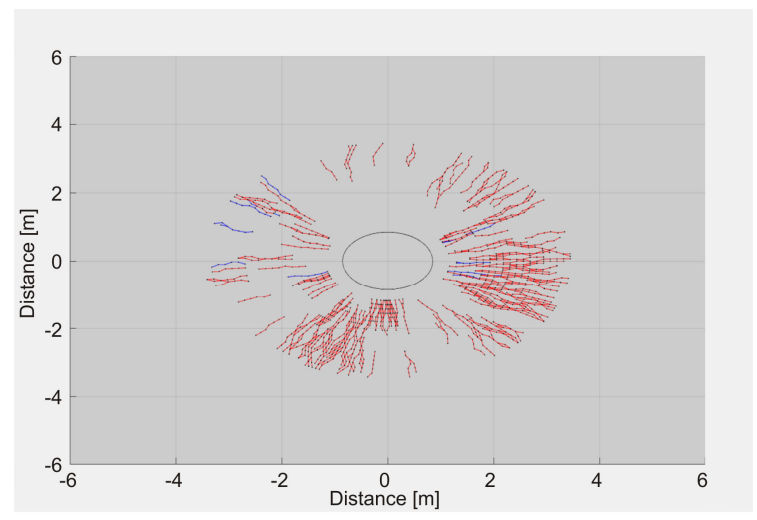

(a)

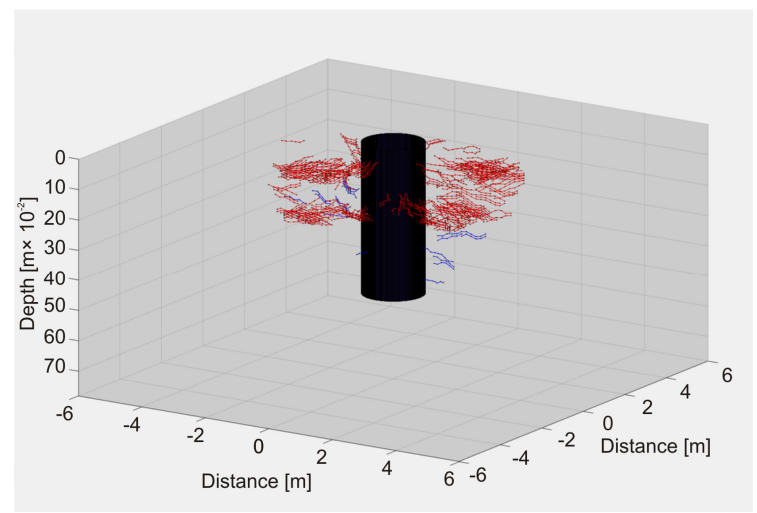

(b)

Fig. 5. Application of the tree root tracking algorithm to the data collected in the fir tree survey (shallow roots in red; deep roots in blue). 2-D plan view (a) and 3-D rendering (b) of the tree root system. 


\section{B. Oak Tree Investigation}

In regard to the application of the pre-processing algorithm to the raw data, it was decided to apply the gain function from the depth of $50 \mathrm{~cm}$ downwards. This was due to the attenuation between $50 \mathrm{~cm}$ and $1 \mathrm{~m}$ that was noticed from the analysis of the raw GPR acquisitions. Fig. 6 shows a comparison between Bscans with application of gain function under $1 \mathrm{~m}$ (Fig. 6(a)) and under $0.5 \mathrm{~m}$ (Fig. 6(b)). As can be seen from the common rectangular windows, the gain function improved the visualisation of the reflection hyperbolas when gain was applied from $0.5 \mathrm{~m}$ downwards. This processing has allowed to pinpoint more targets to connect in the subsequent tree root tracking algorithm stage.

Fig. 7 shows the outputs of the root tracking algorithm. From the plan view in Fig. 7(a), it can be seen that the continuous segments were compatible with the radial expansion of the roots of the examined tree. However, the development of these segments was limited in depth, as only a small amount of deep roots was identified. This result was associated with a likely high humidity of the soil and subsurface water-saturated areas far from the ground surface due to recent and continuous rains. Fig. 7(b) shows the 3-D rendering after the application of the "Evaluation of root diameters" stage of the tracking algorithm. In this regard, the minimum detectable diameter (i.e. the minimum distance between two adjacent points along the $z$ axis) was strictly related to spatio-temporal relationship between the vertical (time) resolution of the GPR signal $\left(d t=7.812510^{-11} \mathrm{~s}\right)$ and the average value of velocity of propagation calculated from hyperbola fitting. This turned out to provide a minimum value of detectable roots $\sim 8 \mathrm{~cm}$. Comparing number of roots in Fig. 7(a)-(b), it can be seen that the majority have been displayed in the 3-D rendering, meaning that few fine roots have been detected.

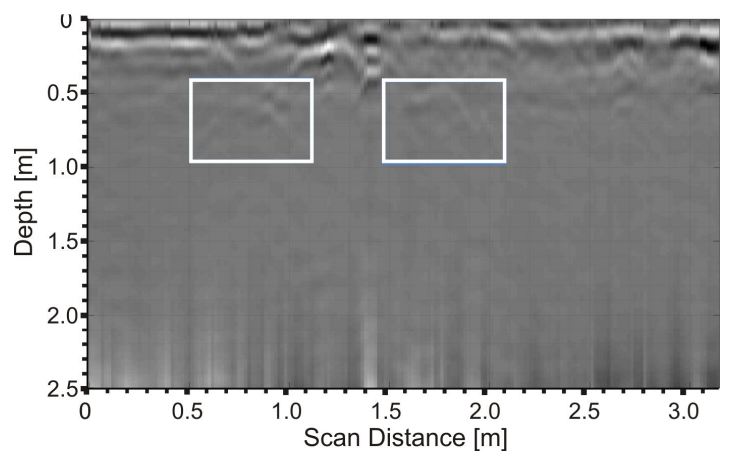

(a)

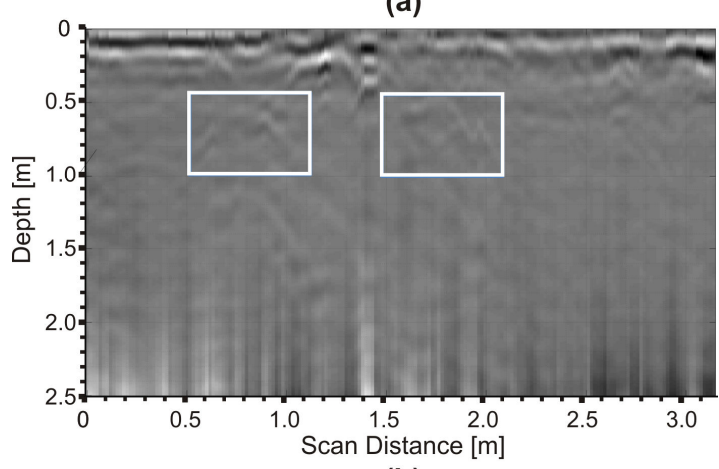

(b)

Fig. 6. Comparison between B-scans after the application of gain function under $1 \mathrm{~m} \mathrm{(a)}$ and under $0.5 \mathrm{~m}(\mathrm{~b})$.

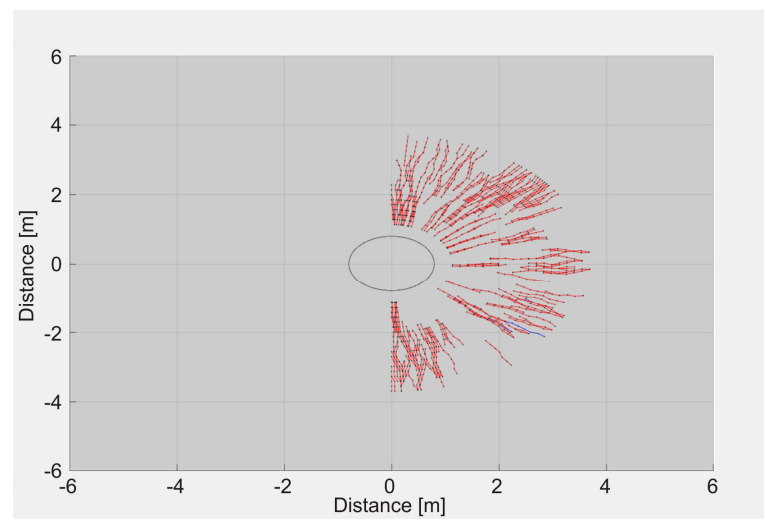

(a)

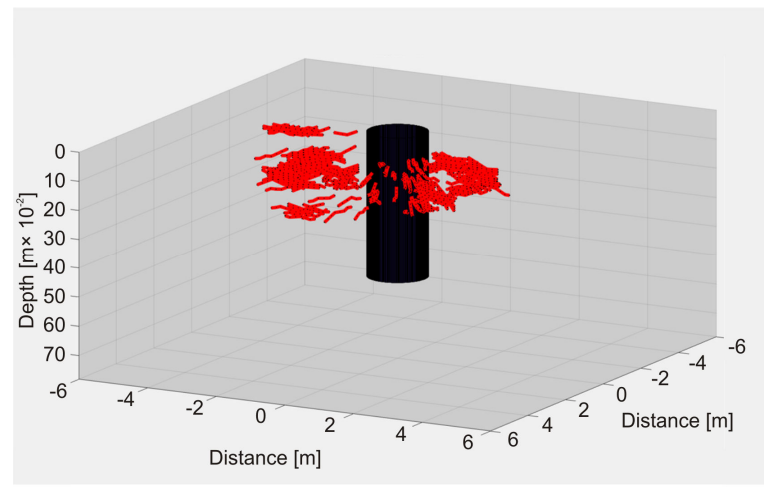

(b)

Fig. 7. Application of the tree root tracking algorithm to the data collected in the oak tree survey (shallow roots in red; deep roots in blue). 2-D plan view of the tree root system (a) and 3-D rendering with evaluation of diameters (b).

\section{CONCLUSION}

This study reports a demonstration of the ground penetrating radar (GPR)'s potential in the health monitoring of tree roots. The main aim of the research was to provide effective and highresolution mapping of tree root systems.

To this purpose, a dedicated data processing methodology, based on two main chronological stages, was developed. An algorithm was firstly proposed to filter out the data from noiserelated information and to enhance deep reflections from attenuated targets. Subsequently, a multi-step algorithm connecting the identified targets (i.e. the vertices of the reflection hyperbolas) in a three-dimensional environment was created.

To demonstrate the viability of the proposed methodology, the soils around two different tree species (i.e. fir and oak trees) were investigated using a ground-coupled multi-frequency GPR system equipped with $600 \mathrm{MHz}$ and $1600 \mathrm{MHz}$ central frequency antennas.

The results have proven the capabilities of the proposed algorithm in identifying shallow (i.e. within the first $25 \mathrm{~cm}$ from the soil surface) and deep (i.e. below $25 \mathrm{~cm}$ from the soil surface) tree root systems for different species of trees. In addition, the potential for the assessment of tree roots with a measurable thickness (i.e. roots with a size that exceeded the minimum vertical resolution of the GPR signal) was demonstrated. 


\section{ACKNOWLEDGMENTS}

The authors would like to express their sincere thanks and gratitude to the following trusts, charities, organisations and individuals for their generosity in supporting this project: Lord Faringdon Charitable Trust, The Schroder Foundation, Cazenove Charitable Trust, Ernest Cook Trust, Sir Henry Keswick, Ian Bond, P. F. Charitable Trust, Prospect Investment Management Limited, The Adrian Swire Charitable Trust, The John Swire 1989 Charitable Trust, The Sackler Trust, The Tanlaw Foundation, and The Wyfold Charitable Trust.

The authors would also like to acknowledge Mr. Spartaco Cera for the technical assistance provided during the GPR surveys.

\section{REFERENCES}

[1] S.D. Day, P. E. Wiseman, S. B. Dickinson, and J.R. Harris, "Contemporary concepts of root system architecture of urban trees," Arbor. Urb. For., vol. 36, pp. 149-159, 2010

[2] S. J. Pallardy, Physiology of woody plants. Academic Press, 2010.

[3] W.H. Lyford, and B.F. Wilson, Development of the root system of Acer Rubrum, In Harvard Forest. Petersham, Massachusetts: Harvard University, 1964.

[4] B.F. Wilson, Structure and growth of woody roots of Acer Rubrum L. In Harvard Forest. Petersham, Massachusetts: Harvard University, 1964

[5] A. Stokes, A.H. Fitter and M.P. Coutts, "Responses of young trees to wind and shading: effects on root architecture and anchorage strenght," J. Exp. Bot., vol. 46, pp. 1139-1146, 1995.

[6] K.S. Pregitzer, M.J. Laskowski, A.J. Burton, V.C. Lessard, and D.R. Zak, "Variation in sugar maple root respiration with root diameter and soil depth,” Tree Physiol., vol. 18, pp. 665-670, 1998.

[7] K.S. Pregitzer, J. L. DeForest, A.J. Burton, M.F. Allen, R.W.Ruess, and R.L. Hendrick, (2002). Fine root architecture of nine North American trees. Ecological Monographs, 72, 293-309.

[8] J. Roberts, (1976). A study of root distribution and growth in a Pinus Sylvestris L. (Scots Pine) plantation in East Anglia. Plant and Soil, 44, 607-621.

[9] A.G. Bengough, B.M. McKenzie, P.D. Hallett, \& T.A. Valentine, (2010). Root elongation, water stress, and mechanical impedance: a review of limiting stresses and beneficial root tip traits. Journal of experimental botany, 62(1), 59-68.

[10] J.A., Franco, S., Banon, M.J. Vicente, J. Miralles, \& J.J. MartinezSanchez, (2011). Root development in horticultural plants grown under abiotic stress conditions - a review. The Journal of Horticultural Science and Biotechnology, 86(6), 543-556.

[11] A.L., Smit, A.G. Bengough, C. Engels, M. Van Noordwijk, S. Pellerin, \& v.d. Geijn, (2013). Root Methods: A Handbook. Springer Science \& Business Media.

[12] S. Lambot, L. Weihermüller, J.A. Huisman, H. Vereecken, M. Vanclooster and E.C. Slob, 2006. Analysis of air-launched groundpenetrating radar techniques to measure the soil surface water content. Water Resources Research 42(11): W11403. doi: 10.1029/2006WR005097.

[13] A. Benedetto, F. Tosti, B. Ortuani, M. Giudici, and M. Mele, "Mapping the spatial variation of soil moisture at the large scale using GPR for pavement applications," Near Surface Geophysics, vol. 13 (3), pp. 269$278,2005$.

[14] A. Benedetto, F. Tosti, B. Ortuani, M. Giudici and M. Mele, "Soil moisture mapping using gpr for pavement applications," In: Proc. of the Seventh International Workshop on Advanced Ground Penetrating Radar, Nantes, France, Jul. 2013, pp. 243-248.
[15] F. Tosti and E.C. Slob, "Determination, by using GPR, of the volumetric water content in structures, substructures, foundations and soil," in Civil Engineering Applications of Ground Penetrating Radar (Springer Trans. Civil and Environ. Eng. Book Series), A. Benedetto and L. Pajewski, Eds., Springer International Publishing, pp. 163-194, 2015.

[16] J. Huisman, S. Hubbard, J. Redman, and A. P. Annan, "Measuring soil water content with ground penetrating radar: A review," Vadose Zone J., vol. 2, no. 4, pp. 476-491, 2003.

[17] J.R. Butnor, J.A. Doolittle, L. Kress, S. Cohen, and K.H. Johnsen, 2001. Use of ground-penetrating radar to study tree roots in the southeastern United States. Tree Physiology, 21 (17): 1269-1278.

[18] C.V.M. Barton, K.D. Montagu, Detection of tree roots and determination of root diameters by ground penetrating radar under optimal conditions (2004) Tree Physiology, 24 (12): 1323-1331.

[19] J.R. Butnor, J.A. Doolittle, K.H. Johnsen, L. Samuelson, T. Stokes, and L. Kress, Utility of ground-penetrating radar as a root biomass survey tool in forest systems (2003) Soil Science Society of America Journal, 67 (5): 1607-1615.

[20] L. Guo, J. Chen, X. Cui, B. Fan, H. Lin, Application of ground penetrating radar for coarse root detection and quantification: A review (2013) Plant and Soil, 362 (1-2), pp. 1-23.

[21] A. Stokes, T. Fourcaud, J. Hruska, J. Cermak, N. Nadyezdhina, V. Nadyezhdin, and L. Praus, An evaluation of different methods to investigate root system architecture of urban trees in situ: I. groundpenetrating radar (2002) Journal of Arboriculture, 28 (1): 2-10.

[22] F. Tosti, L. Bianchini Ciampoli, M.G. Brancadoro, and A.M. Alani, (2018). GPR applications in tracking the subsurface root system of street trees with road safety-critical implications. Advances in Transportation Studies - An International Journal. (Submitted).

[23] J.R. Butnor, M.L. Pruyn, D.C. Shaw, M.E. Harmon, A.N. Mucciardi, and M.G. Ryan, 2009. Detecting defects in conifers with ground penetrating radar: applications and challenges, Forest Pathol., 39 (5): 309-322.

[24] R. Martínez-Sala, I. Rodríguez-Abad, R. Diez Barra, and R. CapuzLladró, 2013. Assessment of the dielectric anisotropy in timber using the nondestructive GPR technique, Constr. Build. Mater., 38: 903-911.

[25] I. Rodríguez-Abad, R. Martínez-Sala, F. García-García, and R. CapuzLladro, 2010. Nondestructive methodologies for the evaluation of moisture content in sawn timber structures: ground-penetrating radar and ultrasound techniques, Near Surf. Geophys. 8: 475-482.

[26] H. Sahin, and N. Ay, 2004. Dielectric properties of hardwood species at microwave frequencies, J. Wood Sci., 50 (4): 375-380.

[27] J. Ježová, L. Mertens, and S. Lambot, 2016. Ground-penetrating radar for observing tree trunks and other cylindrical objects, Construction and Building Materials, 123: 214-225.

[28] A.M. Alani, L. Bianchini Ciampoli, F. Tosti, M.G. Brancadoro, D. Pirrone, and A. Benedetto (2017). Health monitoring of a matured tree using ground penetrating radar - investigation of the tree root system and soil interaction. In: Proc. of the IMEKO International Conference on Metrology for Archaeology and Cultural Heritage (MetroArchaeo2017), October 23-25, 2017, Lecce, Italy.

[29] A. Benedetto, F. Tosti, L. Bianchini Ciampoli, and F. D'Amico, (2017). An overview of ground-penetrating radar signal processing techniques. Signal processing, 132, 201-209.

[30] D.J. Daniels, Ground Penetrating Radar. The Institution of Electrical Engineers, London, 2004.

[31] H. Jol, Ground Penetrating Radar: Theory and Applications, Elsevier, Amsterdam, The Netherlands, 2009.

[32] G. Olhoeft, (2000). Maximizing the information return from ground penetrating radar, Journal of Applied Geophysics, 43, pp. 175-187.

[33] S. W. Yeung, W.M. Yan, and C.H. Hau, (2016). Performance of ground penetrating radar in root detection and its application in root diameter estimation under controlled conditions. SCIENCE CINA Earth Sciences, 59(1), 145-155. 